\title{
Variación de los hábitos tróficos en nueve especies de peces de la Laguna Garzón (Uruguay)
}

\section{Feeding habits variation within nine fish species from Laguna Garzón (Uruguay)}

García-Spósito, Camilo (1); Malfatti, Francisco (1); Laborde, Sabrina (1); Scarabino, Fabrizio (1); García-Rodríguez, Felipe (2); Bergamino, Leandro (1)

(1) Centro Universitario Regional del Este, UdelaR, Rocha, Uruguay.

(2) Instituto Oceanografico, Programa de Pós-Graduação em Oceanografia Física, Química e Geológica, Instituto de Oceanografia, Universidade Federal do Rio Grande, Rio Grande, RS, Brazil.

Contacto: camilogarcia1001@gmail.com

RECIBIDO: $11 / 4 / 2018$ - APROBADO: $31 / 8 / 2018$

\begin{abstract}
Resumen
Se analizó el rol trófico de nueve especies de peces capturadas en la Laguna Garzón (costa atlántica uruguaya) mediante la evaluación de la composición isotópica de nitrógeno y carbono. La captura se realizó en mayo de 2017 utilizando redes de enmalle de $10 \mathrm{~cm}$ y $15 \mathrm{~cm}$ entre nudos opuestos, con una longitud total de $200 \mathrm{~m}$ y un tiempo de actividad de $12 \mathrm{~h}$. Los resultados indicaron una variación isotópica en las especies analizadas debido a diferentes hábitos tróficos. Las mayores posiciones tróficas (PT) ocuparon el segundo nivel trófico, con especies como la anchoa Lycengraulis grossidens $(\mathrm{PT}=3,8)$, el testolín azul Prionotus punctatus $(\mathrm{PT}=3,4)$, la corvina blanca Micropogonias furnieri $(3,4)$, y el dientudo Oligosarcus jenynsii $(\mathrm{PT}=3,0)$. Los modelos isotópicos mostraron que algunas especies estuvieron asociadas al ambiente bentónico, y se alimentaron preferentemente de material orgánico del sedimento (Mugil liza), o de cangrejos (M. furnieri), en contraste con especies que estuvieron asociadas a la columna de agua, como el pejerrey Odontesthes argentinensis. De esta forma, la fauna bentónica residente y la materia orgánica del sedimento y en suspensión estarían sustentando los hábitos de alimentación de los peces analizados en la Laguna Garzón. Palabras clave: variabilidad isotópica, redes tróficas, peces costeros, estuario.
\end{abstract}

\begin{abstract}
Trophic role including nine fish species from a coastal lagoon in Uruguay (Laguna Garzón) was assessed using carbon and nitrogen stable isotope measurements. To this end, samples of food sources and fishes were collected in May 2017 for isotopic analysis. Fishes were collected by deploying a net of 10 and $15 \mathrm{~cm}$ (200 m long) for $12 \mathrm{hs}$. The results indicated an isotopic variation within the fish species examined thus suggesting different trophic habits. The highest trophic positions (TP) were observed for the anchovy Lycengraulis grossidens $(\mathrm{TP}=3,8)$, the bluewing searobin Prionotus punctatus ( $\mathrm{TP}=3,4)$, the whitemouth croaker Micropogonias furnieri $(3,4)$, and dog-tooth characid Oligosarcus jenynsii ( $\mathrm{TP}=3,0)$. Model calculations showed that the main prey for the fish species in Garzón derived from benthic (e.g. M. furnieri, Mugil liza) and pelagic environment (Odontesthes argentinensis). Therefore, benthic fauna together with sedimentary carbon sources support this fish assemblage in Laguna Garzón. Keywords: isotopic variation, food webs, coastal fishes, estuary.
\end{abstract}

\section{Introducción}

Las lagunas costeras se caracterizan por ser ecosistemas altamente productivos, dado el gran aporte de nutrientes que reciben tanto del continente como de los ríos y el mar adyacente, en combinación con una alta disponibilidad lumínica (Knoppers, 1994). Estos sistemas costeros brindan numerosos servicios ecosistémicos para la biodiversidad y para el hombre, entre ellos, el sustento de pesquerías artesanales, sitios de alimentación y reproducción para diversas especies de peces y aves, la recrea- ción y el control de la erosión (Costanza, et al., 1997; Fabiano y Santana, 2006). Sin embargo, a pesar de la relevancia para la conservación que estos sistemas costeros presentan, están siendo significativamente amenazados debido al incremento de asentamientos humanos en estas regiones costeras, lo que configura una amenaza física y ecológica para estos sistemas altamente vulnerables (Conde, et al., 2015). En este contexto, la generación de información acerca de la composición, estructura y funcionamiento de los ecosistemas es muy importante para la toma de decisiones de gestión responsable. 
Uno de los aspectos centrales para la toma de decisiones de gestión es el conocimiento de las relaciones alimenticias entre las especies de un ecosistema. Este tipo de información permite predecir cuáles son las potenciales consecuencias de una medida específica sobre el resto de la comunidad biótica y sus recursos (Polis y Winemmiller, 1996). En las lagunas costeras, algunos de los productores primarios que se han identificado y que sustentan la red trófica incluyen micro y macroalgas, epífitas, material orgánico del sedimento y detritus de origen terrestre (Vizzini y Mazzola, 2003; Pernet, et al., 2012; Park, et al., 2013; Careddu, et al., 2015). Asimismo, García et al. (2007) realizaron un estudio puntual durante la primavera austral sobre los hábitos alimenticios en un ensamblaje de peces y sugieren que los productores primarios autóctonos representan la mayor fuente de alimento. En algunos casos las relaciones alimenticias pueden variar espacial y temporalmente (Kanaya, et al., 2007; Claudino, et al., 2013), y en otros no (Rodríguez-Graña, et al., 2008).

Estudios sobre los hábitos alimenticios, como el análisis de contenido estomacal, proveen información sobre la composición de presas y relaciones tróficas y son difíciles de interpretar en el material ingerido en estado triturado y mezclado. Como complemento, la determinación de isótopos estables se ha transformado en una potente herramienta para definir las relaciones tróficas entre los componentes de la comunidad (Fry, 2007). Este hecho se basa en que las proporciones de isótopos estables de carbono $\left(\delta^{13} \mathrm{C}={ }^{13} \mathrm{C} /{ }^{12} \mathrm{C}\right)$ y nitrógeno $\left(\delta^{15} \mathrm{~N}={ }^{15} \mathrm{~N} /{ }^{14} \mathrm{~N}\right)$ de los animales depende de la ingesta. En general, el $\delta^{13} \mathrm{C}$ en los tejidos de los consumidores se incrementa en $0-1 \%$ respecto de su dieta y, por lo tanto, permite distinguir la fuente de alimentación desde la base del ecosistema (McCutchan, et al., 2003; Caut, et al., 2009). A su vez, el $\delta^{15} \mathrm{~N}$ se estima que incrementa en $2-4 \%$ en el tejido del consumidor respecto al valor de su dieta, y puede ser utilizado para estudiar la posición trófica de un organismo en la cadena trófica (Post, 2002; McCutchan, et al., 2003).

La costa atlántica uruguaya contiene un cordón de lagunas que son ecosistemas con distintos grados de protección y sus pesquerías tradicionales (ej. camarón rosado, Farfantepenaeus paulensis) sustentan asentamientos humanos de escasos recursos (Santana y Fabiano, 1999). En particular, existen trabajos que han detallado los hábitos de alimentación (Norbis y Galli, 2004; Olsson, et al., 2013) y las relaciones alimenticias (Rodríguez-Graña, et al., 2008) en la Laguna de Rocha y se considera importante ampliar este tipo de estudios en el resto de las lagunas costeras. El objetivo de este estudio fue evaluar las variaciones isotópicas de nueve especies de peces capturados en la Laguna Garzón y establecer las principales relaciones energéticas.

\section{Materiales y Métodos}

\section{Área de estudio}

La Laguna Garzón es una laguna somera (profundidad media $=0,6 \mathrm{~m}$; área $=18 \mathrm{~km}^{2}$ ), salobre, que se encuentra dentro de una cuenca de aproximadamente $695 \mathrm{~km}^{2}$, ubicada en la costa sureste de Uruguay $\left(34^{\circ} 46^{\prime} \mathrm{S}, 54^{\circ} 33^{\prime} \mathrm{O}\right.$ ) (Figura 1). Esta laguna presenta una conexión intermitente con el Océano Atlántico, con dos efluentes importantes, el Arroyo Garzón y Arroyo de la Cruz, que desembocan en el NW. La población de habitantes permanente en el área es relativamente baja, los principales usos de esta laguna y su cuenca incluyen la ganadería, forestación, agricultura y urbanización para actividades turísticas, y no se registran actividades industriales (Rodríguez-Gallego, et al., 2017).

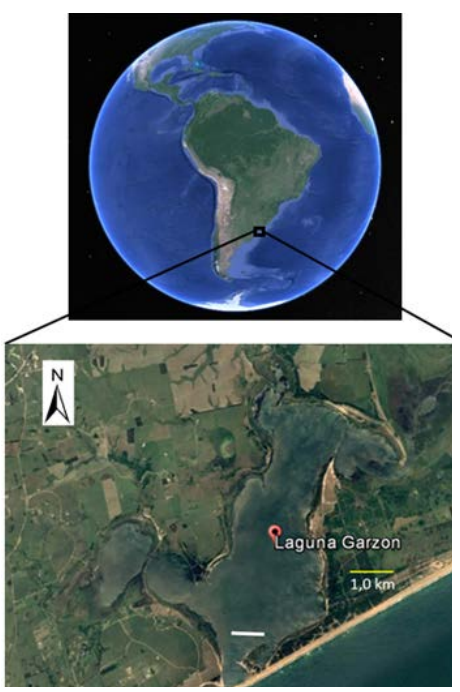

Figura 1. Mapa de la Laguna Garzón (3446’ S, 54³3’ O) en Uruguay. La línea blanca indica la zona en la que fue posicionada la red de enmalle utilizada para el muestreo de peces durante mayo de 2017. Imagen satelital extraída de Google Earth, 20 de marzo de 2017.

\section{Muestreo de peces y fuentes de alimento}

Para la colecta de peces se realizó una sola campaña durante mayo de 2017. La captura se efectuó mediante el calado de una red de enmalle de $10 \mathrm{~cm}$ y $15 \mathrm{~cm}$ de apertura entre nudos opuestos, por una altura de $2 \mathrm{~m}$ y una longitud total de $200 \mathrm{~m}$, durante la noche y por un período de 12 horas, desde las 7:00 PM hasta las 7:00 AM del día siguiente. La zona de calado de la red de enmalle se ubicó en la parte sur de forma perpendicular a la línea de costa (Figura 1). Asimismo, dada la escasa profundidad de estas lagunas se asume que la red abarcó toda la columna de agua. Una vez capturados, los especímenes fueron congelados a una temperatura de $-20^{\circ} \mathrm{C}$ hasta el momento de ser examinados en el laboratorio. Los ejemplares fueron identificados según las claves taxonómicas de Figueiredo y Menezes (2000) y Dyer (2006).

Además, para los análisis isotópicos se colectaron las potenciales fuentes de alimento, incluyendo material orgánico del sedimento (MOS), material orgánico particulado en suspensión $(\mathrm{MOP}=$ fitoplancton + detritus) e invertebrados bentónicos. Para la obtención de MOP se colectó un bidón de $5 \mathrm{~L}$ con agua de la laguna y posteriormente el agua se filtró por triplicado a través de filtros Whatman GF/F almacenándolos hasta su procesamiento a $-20^{\circ} \mathrm{C}$. El MOS se colectó usando un cilindro de PVC con $40 \mathrm{~cm}$ de profundidad y $27 \mathrm{~cm}$ de diámetro y se seleccionó el primer $\mathrm{cm}$ de sedimento para análisis isotópico. Posteriormente, el sedimento fue tamizado a través de una malla de $0,5 \mathrm{~mm}$ y los organismos retenidos se separaron por especie (mínimo cinco ejemplares vivos por especie) y se los congeló para su posterior procesamiento de análisis isotópico. El MOS se compone de fitobentos, bacterias y detritus de diferente origen. Se colectaron tres muestras del sedimento en la zona de muestreo, que posteriormente se almacenaron congeladas a $-20^{\circ} \mathrm{C}$ en frascos plásticos sin uso previo.

\section{Parámetros fisicoquímicos}

Las variables físicoquímicas del agua (temperatura, $\mathrm{pH}, \%$ de oxígeno disuelto), se registraron in situ con un sensor 
multiparámetro Horiba y se tomaron muestras de agua para el análisis de clorofila. Para la determinación de clorofila a, en el laboratorio se filtraron $500 \mathrm{~mL}$ de agua con filtros Whatman GF/C, y se realizó una extracción de $24 \mathrm{~h}$ a oscuras con etanol al 96\% (Jespersen y Christoffersen, 1987). Las mediciones se realizaron en un espectrofotómetro UV/ Vis (modelo SP-2100) ubicado en el Centro Universitario Regional del Este-sede Rocha.

\section{Análisis de isótopos estables}

Para el análisis isotópico en peces se tomaron muestras de músculo dorsal, debido a que presentan menor variación isotópica (Pinnegar y Polunin, 1999). Además, se procesaron moluscos, se descartaron las valvas y solo se usó tejido blando, mientras que en crustáceos decápodos se extrajeron muestras de músculo del segmento abdominal, y en poliquetos se utilizó el organismo entero. Los tejidos de los organismos fueron secados a $60^{\circ} \mathrm{C}$ por $24 \mathrm{~h}$, molidos en un mortero de ágata hasta obtener un polvillo que fue finalmente colocado en cápsulas de estaño. Con el propósito de no alterar las medidas isotópicas, no se aplicó la acidificación de muestras (Serrano, et al., 2008). El análisis isotópico en peces fue realizado para cada individuo capturado por especie; se tomaron entre siete y 10 individuos, según su disponibilidad. En moluscos y crustáceos decápodos fueron realizadas tres réplicas que incluyeron entre cinco y ocho organismos de la misma especie.

Las muestras homogeneizadas preparadas para la medición isotópica de carbono y nitrógeno fueron analizadas por la University of North Carolina-Wilmington (UNCW) mediante la combustión en un analizador elemental Thermo Flash HT Plus. La proporción de isótopos estables de un elemento se expresa a través de la anotación delta $(\delta)$, que representa la diferencia relativa entre las proporciones isotópicas en la muestra y en un estándar (Vienna Pee Dee Belemnite para el carbono y $\mathrm{N}_{2}$ para el nitrógeno), según la Ecuación 1.

$\delta^{13} \mathrm{C}$ o $\delta^{15} \mathrm{~N}(\%$ o $)=\left[\left(\mathrm{R}_{\text {MUESTra }} / \mathrm{R}_{\text {EStÁndar }}\right)-1\right] 1000$

Donde $\mathrm{R}$ es la cantidad de isótopo pesado dividida por la cantidad del ligero, tanto en la muestra como en el estándar: ${ }^{13} \mathrm{C} /{ }^{12} \mathrm{C},{ }^{15} \mathrm{~N} /{ }^{14} \mathrm{~N}$.

\section{Análisis de datos}

La posición trófica (PT) de cada consumidor en la Laguna Garzón se basó en su valor promedio de $\delta^{15} \mathrm{~N}$ para el período de estudio, el cual ha sido demostrado como un indicador confiable de la PT (del Río, et al., 2009). Esta estimación se llevó a cabo siguiendo la fórmula propuesta por Vander Zanden et al. (1997) y modificada por Post (2002):

$\mathrm{PT}_{\mathrm{i}}=\left[\left(\delta^{15} \mathrm{~N}_{\mathrm{i}}-\delta^{15} \mathrm{~N}_{\mathrm{cp}}\right) / 2,9\right]+2$

Donde PTi es la posición trófica promedio del pez i; $\delta^{15} \mathrm{Ni}$ es el promedio de $\delta^{15} \mathrm{~N}$ obtenido del músculo del pez i; $\delta^{15} \mathrm{~N}_{\mathrm{cp}}$ es el promedio de $\delta^{15} \mathrm{~N}$ de los consumidores primarios (en este estudio se considera un promedio entre el caracol Heleobia aff. australis y filtradores Tagelus aff. plebeius); 2,9 corresponde al enriquecimiento trófico promedio de $\delta^{15} \mathrm{~N}$ que ocurre de presa a predador, siguiendo el trabajo de McCutchan et al. (2003), y 2 es la posición trófica de los consumidores primarios. Se consideran organismos base a los consumidores primarios, ya que permiten integrar cambios temporales discontinuos en la base de la red trófica a la vez que abarcan un período de tiempo similar al de los consumidores secundarios (Post, 2002).

Para poder evaluar cuantitativamente la contribución de cada fuente de alimento en la dieta de los peces, se utilizó la aproximación bayesiana de modelos mixtos (SIAR, Stable Isotope Analysis in R; Parnell, et al., 2010). Esta aproximación permite incorporar variabilidad en los parámetros de entrada e incluye los valores isotópicos en consumidores, fuentes de alimento y los factores de enriquecimiento isotópico (músculo en peces: $1,3 \pm 0,13 \%$; para $\delta^{13} \mathrm{C}$ y $2,9 \pm 0,3 \%$ o para $\delta^{15} \mathrm{~N}$; McCutchan, et al., 2003). Para la selección de presas en cada modelo se realizó una revisión bibliográfica de estudios de peces en zonas costeras de Uruguay (Milessi, et al., 2010; Rodríguez-Graña, et al., 2008; Stebniki, 2014; Olsson, et al., 2013). Asimismo, las fuentes de alimento consideradas para cada modelo SIAR debieron formar un polígono de mezcla isotópico que contenga a cada consumidor siguiendo las recomendaciones de Phillips et al. (2014).

\section{Resultados}

\section{Variables ambientales}

En mayo de 2017 se observó que la Laguna Garzón presentó una temperatura de $19^{\circ} \mathrm{C}$, una conductividad de $17,6 \mathrm{mS} / \mathrm{cm}$ y un $\mathrm{pH}$ relativamente neutro, con un valor de 7,9. Asimismo, el porcentaje de saturación de oxígeno disuelto en el agua fue 97,2, y el total de sólidos disueltos 10,9 mg/L. El análisis de clorofila a en el agua fue un promedio de $6,11 \mu \mathrm{g} / \mathrm{L}$.

\section{Caracterización isotópica de los peces analizados}

Se capturaron un total de nueve especies de peces (Tabla 1) y para las especies Odontesthes argentinensis y Mugil liza se identificaron dos grupos de tallas diferentes: mayores a $30 \mathrm{~cm}$ y menores a $10 \mathrm{~cm}$.

\begin{tabular}{|l|c|c|}
\hline Especie & $\mathbf{n}$ & Tamaños (cm) \\
\hline Lycengraulis grossidens & 4 & $15,3(14-16)$ \\
Mugil liza J & 6 & $4,3(4-7)$ \\
Mugil liza A & 5 & $31,8(30-35)$ \\
Micropogonias furnieri & 7 & $23,8(23-25)$ \\
Oligosarcus jenynsii & 5 & $19,2(18-20)$ \\
Cyphocharax voga & 3 & - \\
Cynoscion guatucupa & 7 & - \\
Odontesthes argentinensis J & 4 & $4,5(4-6)$ \\
Odontesthes argentinensis A & 10 & $17,7(15-20)$ \\
Brevoortia aurea & 3 & $12,6(11-14)$ \\
Prionotus punctatus & 3 & $20,5(19-22)$ \\
\hline
\end{tabular}

Tabla 1. Valores promedio de tamaños para cada especie colectada en la Laguna Garzón en mayo de 2017. En paréntesis se indica el rango de tamaños encontrado. n: número de especímenes colectados; J: juveniles; A: Adultos. 


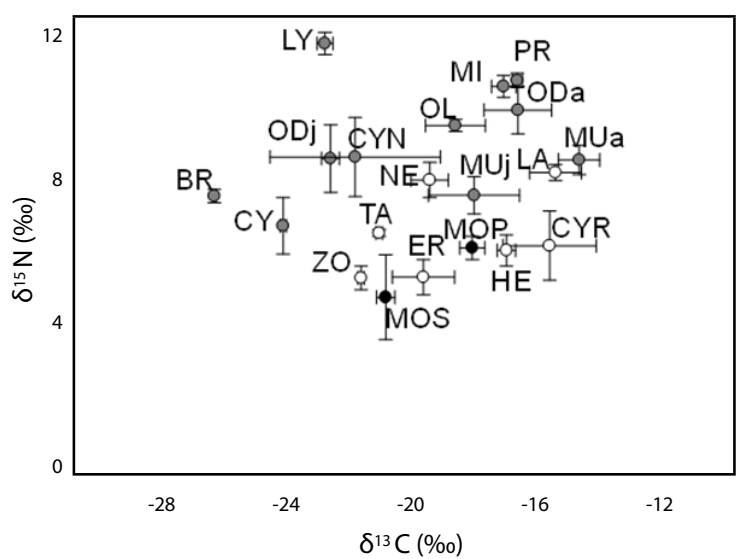

Figura 2. Valores medios ( \pm desvío estándar) de $\delta^{13} \mathrm{C}$ y $\delta^{15} \mathrm{~N}$, en fuentes de materia orgánica (círculos negros), consumidores primarios (círculos blancos) y peces (círculos grises), colectados en la Laguna Garzón en mayo de 2017. MOP: material orgánico particulado; MOS: material orgánico del sedimento; ZO: Zooplancton; ER: Erodona mactroides; LA: Laeonereis pandoensis; HE: Heleobia aff. australis; NE: Nephtys fluviatilis; TA: Tagelus aff. plebeius; ODj; juveniles de Odontesthes argentinensis; MUj: juveniles de Mugil liza; MUa: adultos Mugil liza; BR: Brevoortia aurea; CYR: Cyrtograpsus angulatus; MI: Micropogonias furnieri; PR: Prionotus punctatus; CY: Cyphocharax voga; CYN: Cynoscion guatucupa; LY: Lycengraulis grossidens; OL: Oligosarcus jenynsii.

Los valores de $\delta^{13} \mathrm{C}$ en los peces mostraron una gran variación entre las especies colectadas. La lacha (Brevoortia aurea) y el sabalito (Cyphocharax voga) mostraron los valores más bajos (desde $-26,23 \pm 0,06 \%$ a $-24,09 \pm 0,1 \%$, respectivamente), mientras que los juveniles de la lisa (Mugil liza) y los adultos de pejerrey (Odontesthes argentinensis) presentaron los valores más altos (desde -18,05 $\pm 1,4 \%$ y $-16,80 \pm 0,05 \%$ respectivamente) (Figura 2). Los valores de $\delta^{15} \mathrm{~N}$ mostraron un rango de $7,89 \pm 0,04 \%$ (C. voga) a 12,2 $\pm 0,03 \%$ (anchoa, Lycengraulis grossidens) (Figura 2).

\section{Determinación \\ de las posiciones tróficas}

La PT media más alta en la Laguna Garzón estuvo ocupada por la anchoa (Lycengraulis grossidens), seguida por el testolín azul (Prionotus punctatus) y la corvina blanca (Micropogonias furnieri) ( $\mathrm{PT}=3,85,3,42,3,40$, respectivamente) (Figura 3). El sabalito (Cyphocharax voga) mostró valores de $\delta^{15} \mathrm{~N}$ correspondientes a una $\mathrm{PT}$ de un consumidor primario $(2,1)$, mientras que los juveniles de $M$. liza y $B$. aurea tuvieron valores similares (Figura 3). Asimismo, adultos de $M$. liza, O. argentinensis, la pescadilla (Cynoscion guatucupa) y el dientudo (Oligosarcus jenynsii) tuvieron valores $\delta^{15} \mathrm{~N}$ correspondientes a una PT intermedia entre consumidores primarios y predadores topes (Figura 3).

\section{Importancia de fuentes de alimento en la dieta de peces}

Las estimaciones de los modelos de mezcla isotópicos en la Laguna Garzón identificaron una variación en la contribución relativa de las diferentes fuentes de alimento en la dieta de

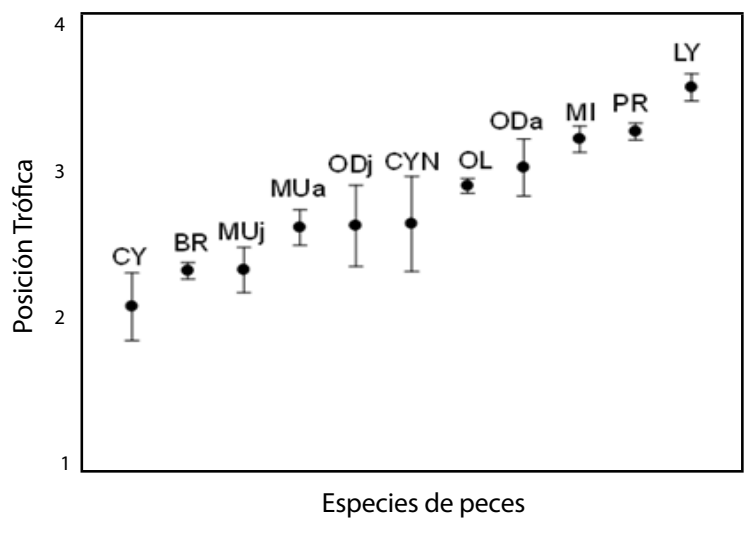

Figura 3. Estimación de las posiciones tróficas a partir de los valores de $\delta^{15} \mathrm{~N}$ en músculo, para las especies de peces colectadas en la zona sur de la Laguna Garzón en mayo de 2017. Las abreviaciones de las especies se muestran en la Figura 2.

los peces (Figura 4). En este sentido, Micropogonias furnieri seleccionó principalmente cangrejos Cyrtograpsus angulatus, con una contribución media en su dieta de 50\%. Asimismo, el caracol Heleobia aff. australis fue la fuente principal de alimento en adultos de M. liza, mientras que para los juveniles de esta especie, el MOS representó la mayor parte de su dieta, con una contribución media de $60 \%$. En individuos adultos de $O$. argentinensis los modelos isotópicos identificaron que poliquetos Nephtys fluviatilis y MOP de la columna de agua fueron los principales componentes dietarios, mientras que para juveniles de pejerrey el consumo de poliquetos disminuyó y aumentó el consumo de zooplancton.

\section{Discusión}

Este estudio mostró una variabilidad en la composición isotópica para las nueve especies de peces capturados en la Laguna Garzón, lo que evidencia la presencia de diferentes hábitos alimenticios y de fuentes de materia orgánica presentes en el sistema. Debido a que las diversas fuentes basales de alimento mostraron valores isotópicos distintos, fue posible la identificación de las vías de transferencia en la red trófica. Se observó que existen vías de transferencia de energía bentónicas y pelágicas hacia los niveles tróficos superiores. Asimismo, los hábitos alimenticios en pejerrey $O$. argentinensis cambiaron con la ontogenia; los individuos juveniles están asociados a hábitos planctónicos, mientras que los organismos adultos estuvieron asociados predominantemente al sistema bentónico en términos dietarios y consumiendo preferentemente poliquetos. Estos resultados están en línea con los mostrados por Stebniki (2014) en estuarios a lo largo de la costa del Río de la Plata, donde tallas menores de O. argentinensis presentaron una alimentación asociada más planctónica que las tallas mayores. Asimismo, la dieta de M. liza también mostró variaciones en la ontogenia con un mayor consumo de Heleobia aff. australis en adultos, mientras que juveniles estuvieron más asociados al material orgánico del sedimento. Esta especie $M$. liza se ha definido como iliófaga, detritívora y capaz de ingerir mayormente diatomeas (Olivera y Soares 1996; Claudino, et al., 2013), y, en consecuencia, caracterizada con una posición 

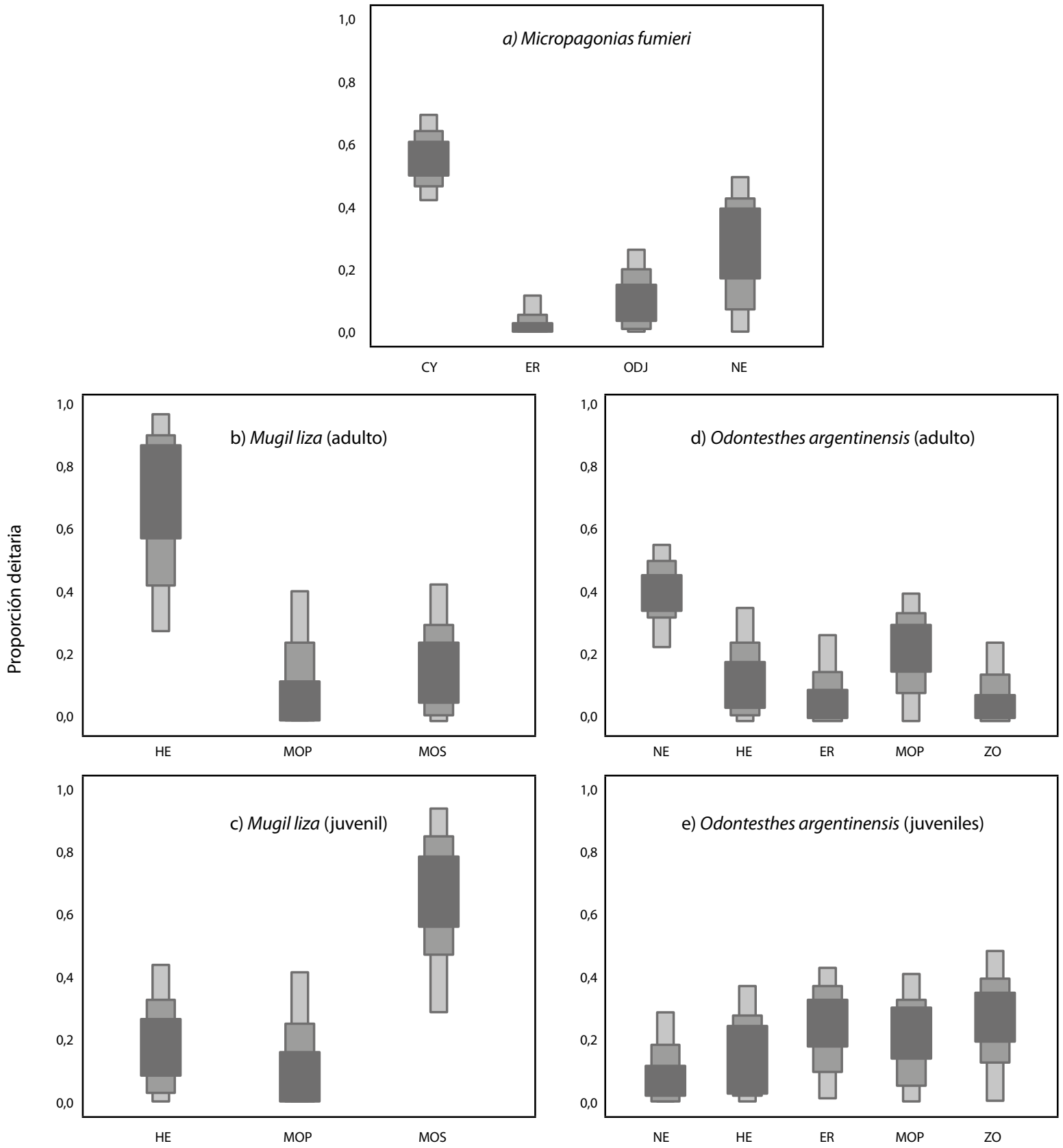

Fuentes de alimento

Figura 4. Resultados de los modelos mixtos isotópicos (SIAR) en la Laguna Garzón, mostrando las estimaciones de las contribuciones de cada fuente de alimento en la dieta de peces Micropogonias furnieri (a), adultos y juveniles Mugil liza (b, c, respectivamente), adultos y juveniles Odontesthes argentinensis ( $d$, e, respectivamente). Las abreviaciones de las fuentes de alimento se muestran en la Figura 2. En cada gráfico las cajas (desde más oscura a más clara) representan los intervalos de credibilidad al 50, 75 y $95 \%$ de la media estimada. Los factores de fraccionamiento aplicados fueron: $\delta 13 \mathrm{C}=1,3 \pm$ $0,13 \%$ y $\delta 15 \mathrm{~N}=2,9 \pm 0,3 \%$ (McCutchan, et al., 2003).

trófica de consumidor primario, tal como se ha encontrado en organismos juveniles de $M$. liza en este trabajo. Pequeños organismos asociados al sedimento como microcrustáceos, anfípodos, bivalvos y gasterópodos también han sido reportados en la dieta de $M$. liza, en línea con los resultados isotópicos obtenidos en este trabajo (Thompson, et al., 2015).

Las fuentes de materia orgánica colectadas en la Laguna Garzón incluyeron el MOP en suspensión (el cual integra al material vivo del fitoplancton y al detritus), MOS y plantas vasculares. Dado que los valores isotópicos de $\delta^{13} \mathrm{C}$ fueron similares entre las especies de peces y las fuentes basales, los resultados de este trabajo indican que existe una transferencia de energía desde las fuentes basales de materia orgánica de la laguna hacia los consumidores, entre ellos, las diversas especies de peces. Estos resultados sugieren que los peces capturados permanecen un tiempo de residencia en el área de estudio, suficiente para que las fuentes de alimento sean transferidas hacia los consumidores. Sin embargo, en la 
lacha B. aurea y el sabalito C. voga se observaron valores isotópicos distintos y separados en la vía de transferencia desde MOP y MOS de la laguna. Esto podría deberse a que la principal fuente de materia orgánica en estos organismos no fue colectada en este estudio y amerita más esfuerzos de muestreo que incluyan el perifiton, o bien estas especies poseen tiempos de residencia menores en Garzón en comparación con el resto de las especies. En el caso de C. voga se ha identificado en arroyos templados un alto porcentaje de material vegetal en su dieta (González-Bergonzoni, 2011). Además, el fuerte vínculo entre consumidores y las fuentes del MOP originado en la columna de agua de la laguna sugiere una contribución trófica de producción autóctona hacia los niveles tróficos superiores, en concordancia con otros estudios en lagunas costeras (García, et al., 2007; Franca, et al., 2011; Prado, et al., 2014).

Las estimaciones de las PT de peces a partir de los datos isotópicos mostraron un máximo de 3,8 , representando el segundo nivel trófico ocupado por la anchoa (Lycengraulis grossidens), seguido de la corvina blanca (Micropogonias furnieri), el testolín azul (Prionotus punctatus) y el dientudo (Oligosarcus jenynsii), lo cual está dentro del rango que describe Milessi et al. (2010) para los peces que habitan en la Laguna de Rocha. Además, estos resultados son corroborados por estudios previos de dieta, como en adultos de anchoa $L$. grossidens reconocidos piscívoros que habitan ambientes marinos, estuarinos y de agua dulce (Mai y Veira, 2013) y en adultos de P. punctatus alimentándose generalmente de organismos bentónicos como cangrejos y camarones (Soares et al., 1998). Se identificaron como consumidores primarios con posiciones tróficas entre dos a tres especies como $M$. liza, B. aurea y juveniles de pejerrey $O$. argentinensis, lo cual concuerda con otros trabajos que identifican material orgánico particulado y zooplancton como principales componentes en sus dietas (Stebniki, 2014). Los modelos SIAR mostraron que en la corvina blanca organismos bentónicos como los cangrejos Cyrtograpsus angulatus fueron los dominantes en su dieta. Estos resultados están en línea con los del trabajo de Olsson et al. (2013), quienes realizaron evaluaciones en contenido estomacal de corvinas de la laguna de Rocha (Uruguay). En el caso de la lisa $M$. liza, el material orgánico del sedimento presentó una alta contribución en la dieta, como se ha mostrado en otros trabajos de dieta en lagunas costeras (Rodríguez-Graña, et al., 2008; Claudino, et al., 2013). Por otra parte, se observó que los juveniles de pejerrey mostraron un mayor consumo sobre zooplancton, mientras que los adultos consumieron mayoritariamente poliquetos. Este cambio en el hábito trófico en pejerrey ha sido observado en otros ambientes estuarinos y podría deberse a cambios morfológicos en la posición de la boca (Bemvenuti, 2006; Stebniki, 2014).

Los bajos valores de conductividad registrados en este trabajo son característicos de sistemas salobres y similares a los encontrados en Laguna Garzón y de Rocha durante los meses de mayores precipitaciones (Conde, et al., 2000; Giménez, et al., 2014; Rodríguez-Gallego, et al., 2017). Durante el muestreo aquí reportado la Laguna Garzón presentaba cerrada la conexión con el océano desde diciembre de 2016 (Verónica Pombo, comunicación personal) impidiendo la intrusión marina y aumentando los tiempos de residencia del agua en la laguna. En este contexto, en la Laguna Garzón la apertura de la barra arenosa en los meses de invierno, frecuentemente en julio, podría cambiar la composición y estructura del ensamblaje de peces por mezcla y reemplazo de aguas (ej. Saad, et al., 2002), que deberían investigarse en futuros estudios.
Este trabajo mostró la diversidad de hábitos alimenticios en nueve especies de peces capturados en la Laguna Garzón, los cuales incluyen: 1) vías planctónicas, alimentadas de material orgánico particulado, como en el caso de pejerrey O. argentinensis, y 2) vías bentónicas, alimentadas de cangrejos y poliquetos, como la corvina blanca. En momentos en que la conexión de la laguna con el océano adyacente está cerrada, el tiempo de residencia de estos organismos parecería ser suficiente para asimilar la fuente de alimento basal del sistema. Este hecho acentúa el rol ecosistémico de la Laguna Garzón como ambiente de alimentación para las especies analizadas.

\section{Reconocimientos}

Los autores agradecen a Soledad Ghione, Verónica Pombo, Héctor Caymaris y Daniel Sosa del SNAP por el apoyo logístico en los muestreos. También agradecemos a Wilson Sebastián Serra (Centro Universitario Regional del Este, sede Rocha) por la ayuda en el reconocimiento taxonómico de las especies de peces. A los pescadores locales "Beto" y "Ñeca", quienes nos permitieron utilizar sus herramientas de trabajo (bote y redes) para la colecta de peces y hacer de su casa una estación de trabajo. A la Comisión Sectorial de Investigación Científica (CSIC), que brindó apoyo financiero por medio de los fondos PAIE y proyecto CSIC I+D 2016 N$^{\circ} 13$, al PEDECIBA-Geociencias y al SNI-ANII.

\section{Referencias}

Bemvenuti, M., 2006. Silversides in South Brazil: morphological and ecological aspects. En: Biocell, 30, pp.111-118.

Careddu, G., Costantini, M., Calizza, E., Carlino, P., Bentivoglio, F., Orlandi, L. y Rossi, L., 2015. Effects of terrestrial input on macrobenthic food webs of coastal sea are detected by stable isotope analysis in Gaeta Gulf. En: Estuarine, Coastal and Shelf Science, 154, pp.158-168.

Caut, S., Angulo, E. y Courchamp, F., 2009. Variation in discrimination factors $\left(\delta^{15} \mathrm{~N}\right.$ and $\left.\delta^{13} \mathrm{C}\right)$ : the effect of diet isotopic values and applications for diet reconstruction. En: Journal of Applied Ecology, 46, pp.443-453.

Claudino, M., Abreu,P. y García, A., 2013. Stable isotopes reveal temporal and between-habitat changes in trophic pathways in a southwestern Atlantic estuary. En: Marine Ecology Progress Series, 489, pp.29-42.

Conde, D., Aubriot, L., y Sommaruga, R., 2000. Changes in UV penetration associated with marine intrusions and freshwater discharge in a shallow coastal lagoon of the Southern Atlantic Ocean. En; Marine Ecology Progress Series, 207, pp.19-31.

Conde, D., Vitancurt, J., Rodríguez-Gallego, L., de Álava, D., Verrastro, N., Chreties, C. y Seijo, L., 2015. Solutions for sustainable coastal lagoon management: from conflict to the implementation of a consensual decision tree for artificial opening. En: Baztan Omer, Juan, Chouinard, Bethany, Jorgensen, Paul, Tett, Jean-Paul y Vanderlinden, LietteVasseur. Coastal zones: solutions for the 21st Century. Cap.13. Nueva York: Elsevier.

Costanza, R., d'Arge, R., de Groot, R., Farber, S., Grasso, M., Hannon, B., Limburg, K., Naeem, S., O’Neill, R., Paruelo, J., Raskin, R., Sutton, P. y van den Belt, M., 1997. The value 
of the world's ecosystem services and natural capital. En: Nature, 387, pp.253-260.

del Río, M., Wolf, N., Carleton, S. y Gannes, L., 2009. Isotopic ecology ten years after a call for more laboratory experiments. En: Biological Reviews, 84, pp.91-111.

Dyer, B., 2006. Systematic revision of the South American silversides (Teleostei, Atheriniformes). En: Biocell, 30, pp. 69-88.

Fabiano, G. y Santana, O., 2006. Las pesquerías en las lagunas salobres de Uruguay. En: Menafra, R., et al.,eds. Bases para la conservación y el manejo de la costa uruguaya. Montevideo: Vida Silvestre Uruguay. pp. 557-565.

Figueiredo, J. y Menezes, N., 2000. Manual de peixes marinhos do sudeste de Brasil VI. San Pablo: Universidad de San Pablo

Franca, S., Vasconcelos, S., Tanner, C., Maguas, M., Costa y Cabral, H., 2011. Assessing food web dynamics and relative importance of organic matter sources for fish species in two Portuguese estuaries: A stable isotope approach. En: Marine Environmental Research, 72, pp.204-215.

Fry, B., 2007. Stable isotope ecology. New York: Springer Science.

García, A., Hoeinghaus, D., Vieira, J. y Winemiller, K., 2007. Isotopic variation of fishes in freshwater and estuarine zones of a large subtropical coastal lagoon. En: Estuarine, Coastal and Shelf Science, 73, pp.399-408.

Giménez, L., Venturini, N., Kandratavicius, N., Hutton, M., Lanfranconi, A., Rodriguez, M., Brugnoli, E., y Muniz, P., 2014. Macrofaunal patterns and animal- esediment relationships in Uruguayan estuaries and coastal lagoons (Atlantic coast of South America). En: Journal of Sea Research, 87, pp.46-55.

González-Bergonzoni, I., 2011. Dieta de peces de agua dulce: efectos de factores climáticos y complejidad del hábitat. Montevideo: Facultad de Ciencias. (Tesis de Maestría), pp. 61

Jespersen, A., Christoffersen, K., 1987. Measurements of chlorophyll-a from phytoplankton using ethanol as extraction solvent. En: Archive of Hydrobiologia, 109, pp.445-454.

Kanaya, G., Takagi, S., Nobat, E. y Kikuchi, E., 2007. Spatial dietary shift of macro- zoobenthos in a brackish lagoon revealed by carbon and nitrogen stable isotope ratios. En: Marine Ecology Progress Series, 345, pp.117-127.

Knoppers, B., 1994. Aquatic primary production in coastal lagoons. En: Kjerfve, B., ed. Coastal lagoon processes. Amsterdam: Elsevier.

Mai, A. y Veira, J., 2013. Review and consideration on habitat use, distribution and life history of Lycengraulis grossidens (Agassiz, 1829) (Actinopterygii, Clupeiformes, Engraulididae). En: Biota Neotrop., 13, pp.121-130.

McCutchan, J., Lewis, W., Kendall, C. y McGrath, C., 2003. Variation in trophic shift for stable isotope ratios of carbon, nitrogen, and sulfur. En: Oikos, 102, pp.378- 390.

Milessi, A., Calliari, D., Rodríguez, L., Conde D., Sellanes, J. y Rodríguez-Gallego, L., 2010. Trophic mass-balance mode of a subtropical coastal lagoon, including a comparison with a stable isotope analysis of the food-web. En: Ecological Modelling, 221, pp.2859-2869.

Norbis, W. y Galli, O., 2004. Feeding habits of the flounder Paralichthysorbignyanus (Valenciennes 1839) in a shallow coastal lagoon of the southern Atlantic Ocean: Rocha, Uruguay. En: Ciencias Marinas, 30, pp.619-625.
Oliveira, I. y Soares, L., 1996. Alimentacao da tainha Mugil platanus Günther, 1880 (Pisces: Mugilidae), da região estuarino-lagunar de Cananéia, São Paulo, Brasil. En: Bol. Inst. Pesca, 23, pp.95-104.

Olsson, D., Forni, F., Saona, G., Verocai, J. y Norbis, W., 2013 Temporal feeding habits of the whitemouth croaker Micropogoniasfurnieri in a shallow coastal lagoon (southwestern Atlantic Ocean, Uruguay). En: Ciencias Marinas, 39, pp.265-276.

Park, H., Choy, E., Lee, K. y Kang, C., 2013. Trophic transfer between coastal habitats in a seagrass-dominated macrotidal embayment system as determined by stable isotope and fatty acid signatures. En: Marine and Freshwater Research, 64, pp.1169-1183.

Parnell, A., Inger, R., Bearhop, S. y Jackson, A., 2010. Source partitioning using stable isotopes: Coping with too much variation. En: PLOS One, 5, pp.e9672.

Pernet, F., Malet, N., Pastoureaud, A., Vaquer, A., Quéré, C. y Dubroca, Laurent, 2012. Marine diatoms sustain growth of bivalves in a Mediterranean lagoon. En: Journal of Sea Research, 68, pp.20-32.

Phillips, D., Inger, R., Bearhop, S., Jackson, A., Moore, J., Parnell, A., Semmens, B. y Ward, E., 2014. Best practices for use of stable isotope mixing models in food-web studies. En: Canadian Journal of Zoology, 92, pp.823-835.

Pinnegar, J., y Polunin, N., 1999. Differential fractionation of $\delta 13 \mathrm{C}$ and $\delta 15 \mathrm{~N}$ among fish tissues: implications for the study of trophic interactions. En: Functional Ecology, 13, pp.225-231.

Polis, G. y Winemiller, K., 1996. Food webs: integration of patterns and dynamics. New York: Chapman and Hall

Post, D., 2002. Using stable isotopes to estimate trophic position: models, methods, and assumptions. En: Ecology 83, pp.703-718.

Prado, P., Vergara, C., Caiola, N. e Ibáñez, C., 2014. Influence of salinity regime on the food-web structure and feeding ecology of fish species from Mediterranean coastal lagoons. En: Estuarine, Coastal and Shelf Science, 139, pp.1-10

Rodríguez-Gallego, L., Achkar, M., Defeo, O., Vidal, L., Meerhoff, E. y Conde, D., 2017. Effects of land use changes on eutrophication indicators in five coastal lagoons of the Southwestern Atlantic Ocean. En: Estuarine, Coastal and Shelf Science, 188, pp.116-126.

Rodríguez-Graña, L., Calliari, C., Conde, D., Sellanes, J. y Urrutia, R., 2008. Food web of a SW Atlantic shallow coastal lagoon: spatial environmental variability does not impose substantial changes in the trophic structure. En: Marine Ecology Progress Series, 362, pp.69-83.

Saad, A.M., Beaumord, A.C., y Caramaschi, E.P., 2002. Effects of artificial canal openings on fish community structure of Imboassica Coastal Lagoon, Rio de Janeiro, Brazil. En: Journal of Coastal Research, 36, pp. 634-639.

Santana, O. y Fabiano G., 1999. Medidas y mecanismos de administración de los recursos pesqueros de las lagunas costeras del litoral atlántico del Uruguay (Lagunas de José Ignacio, Garzón, de Rocha y de Castillos). Plan de Investigación Pesquera INAPE-PNUD URU/92/003. Apéndice 1. Montevideo: INAPE, PNUD.

Serrano, O., Serrano, L., Mateo, M., Colombini, I., Chelazzi, L., Gagnarli, E. y Fallaci, M., 2008. Acid washing effect on elemental and isotopic composition of whole beach arthropods: implications for food web studies using stable isotopes. En: Acta Oecologica, 34, pp, 89-96. 
Soares, L.S.H., Jarre-Teichmann, A. y Rosi-Wongtschowski, C.L., 1998. Field estimates of food consumption of the searobin Prionotus punctatus (Bloch, 1797) on the continental shelf off Ubatuba, southeastern Brazil. En: Revista Brasileira de Oceanografía, 46, pp.45-60.

Stebniki, 2014. Hábitos tróficos de cuatro especies de peces en los estuarios Pando, Solís Chico y Solís Grande (Dpto. Canelones, Uruguay). Montevideo: Facultad de Ciencias. (Tesis de Grado), 39 pp.

Thompson G, Callico Fortunato, R., Chiesa, I. y Volpedo, A., 2015. Trophic ecology of Mugil liza at the southern limit of its distribution (Buenos Aires, Argentina). En: Brazilian Journal of Oceanography, 63, pp.271-278.

Vander Zanden, M., Cabana, G. y Rasmussen, J., 1997. Comparing trophic position of freshwater littoral fish species using stable nitrogen isotopes $(\delta 15 \mathrm{~N})$ and literature dietary data. En: Canadian Journal of Fisheries and Aquatic Sciences, 54, pp.1142-1158.

Vizzini, S. y Mazzola, A., 2003. Seasonal variations in the stable carbon and nitrogen isotope ratios $\left({ }^{13} \mathrm{C} /{ }^{12} \mathrm{C}\right.$ and $\left.{ }^{15} \mathrm{~N} /{ }^{14} \mathrm{~N}\right)$ of primary producers and consumers in a western Mediterranean coastal lagoon. En: Marine Biology, 142, pp.1009-1018. 